Eric S. Maskin • Kevin W.S. Roberts

\title{
On the Fundamental Theorems of General Equilibrium
}

Received: $\quad$ /revised version:

\begin{abstract}
We provide simple generalizations of the three principal theorems of general equilibrium (existence of equilibrium and the two welfare theorems). These enable us to (i) establish equilibrium existence in economies where Walras' Law does not always hold and (ii) show that the second welfare theorem follows from a revealed preference argument as simple as that normally used to prove the first welfare theorem.
\end{abstract}

Keywords: Equilibrium $\cdot$ Pareto optimum $\cdot$ Walras Law

JEL classification: B21· C62 $\cdot$ D51· D61

Research support from the NSF is gratefully acknowledged. The first version of this manuscript was written long ago (November 1980), and one of its main results (Theorem 3) has even become standard textbook fare (see Varian 1992, pp 329 and 336). Two substantial changes have been made to that old version: first, a tax economy example has been removed to focus attention on the core results; second, a new section (Section 4) has been added on related literature. We are grateful to the Editor and two referees for their comments.

Eric Maskin

Institute for Advanced Study and Princeton University, Princeton, NJ 08540, USA

E-mail: maskin@ias.edu

Kevin W. Roberts

Department of Economics and Nuffield College, Oxford, UK

E-mail: kevin.roberts@nuffield.ox.ac.uk 


\section{Introduction}

The three fundamental theorems of general equilibrium theory are the propositions that, under appropriate hypotheses, (i) a competitive equilibrium exists; (ii) a competitive equilibrium is Pareto efficient; and (iii) a Pareto efficient allocation can be decentralized as a competitive equilibrium with transfer payments. Of these theorems, assertion (ii) (often called the First Welfare Theorem) is mathematically virtually trivial, whereas the existence and decentralization results are usually considered "deeper.”

In this paper, we will provide a simple generalization of the existence theorem to economies where Walras' Law (which asserts that the value of excess demand is zero) need not be satisfied out of equilibrium. Assertion (iii) (the Second Welfare Theorem) is an almost immediate corollary of this generalization. Our approach makes it clear that, given the existence of equilibrium, the first and second welfare theorems are equally "trivial”; indeed, we show that they can be proved in very similar ways.

We begin in Section 1 by establishing equilibrium existence for a "generalized competitive" mechanism. In Section 2 we apply this result to a "fixed allocation" mechanism. We take up the welfare theorems in Section 3. Section 4 discusses related literature, and Section 5 concludes.

\section{Generalized Competitive Mechanisms}

Let the basic data of the economy be given by the specification of

preferences $\left\{\succsim^{h}\right\}$, endowments $\left\{\omega^{h}\right\}$, and production sets $\left\{Y^{f}\right\}$, where consumers are indexed by $h=1, \ldots H$, and firms by $f=1, \ldots, F$. For all $h$, consumer $h$ 's preference 
ordering $\succsim^{h}$ is defined ${ }^{1}$ over $\mathbb{R}_{+}^{\ell}$, and his endowment $\omega^{h}$ belongs to $\mathbb{R}_{+}^{\ell}$, where $\ell$ is the number of commodities (the assumption that the consumption space is the positive orthant is more restrictive than necessary). For all $f$, firm $f$ 's production set $Y^{f}$ is a subset of $\mathbb{R}^{\ell}$.

A generalized competitive mechanism (GCM) is a rule that, for each vector of prices $p$ (in the unit simplex) and each specification $\left\{y^{f}\right\}$ of production plans by firms (where $y^{f} \in Y^{f}$ for all $f$ ), assigns to each consumer $h$ an income $I^{h}\left(p,\left\{y^{f}\right\}\right)$. One example of a GCM is, of course, the ordinary competitive mechanism, in which

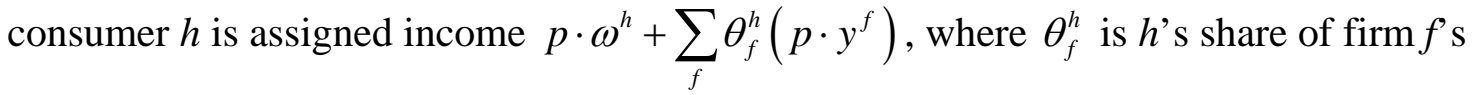
profit $p \cdot y^{f}$, and $\sum_{h} \theta_{f}^{h}=1$ for all $f$. Another example is the mechanism that, given some fixed allocation ${ }^{2}\left\{\left\{\tilde{x}^{h}\right\},\left\{\tilde{y}^{f}\right\}\right\}$ and prices $p$, gives consumer $h$ income $p \cdot \tilde{x}^{h}$.

An equilibrium of a GCM is a price vector $p$ and an allocation $\left\{\left\{x^{h}\right\},\left\{y^{f}\right\}\right\}$ such that (i) for each $h, x^{h}$ is preference-maximizing in $\mathbb{R}_{+}^{\ell}$, subject to the constraint $p \cdot x^{h} \leq I^{h}\left(p,\left\{y^{f}\right\}\right)$; (ii) for each $f, y^{f}$ is profit-maximizing in $Y^{f}$ given prices $p$; and (iii) $\sum_{h} x^{h}=\sum_{f} y^{f}+\sum_{h} \omega^{h}$

${ }^{1}$ As usual, “ $x \succsim^{h} y$ ” means “ $x$ is weakly preferred to $y$ by consumer $h$.”

${ }^{2}$ An allocation is a specification $\left\{x^{h}\right\}$ of consumers' consumption bundles together with a specification $\left\{y^{f}\right\}$ of firms' production plans. The allocation is feasible if $x^{h} \in \mathbb{R}_{+}^{\ell}$ for all $h, y^{f} \in Y^{f}$ for all $f$, and $\sum_{h} x^{h} \leq \sum_{f} y^{f}+\sum_{h} \omega^{h}$. The allocation is Pareto efficient if it is feasible and there is no other feasible allocation $\left\{\left\{\tilde{x}^{h}\right\},\left\{\tilde{y}^{f}\right\}\right\}$ such that $\tilde{x}^{h} \succsim^{h} x^{h}$ for all $h$, with strict preference for some $h$. 
Notice that, in the first example above, the value of excess demand,

$p \cdot\left(\sum_{h}\left(x^{h}-\omega^{h}\right)-\sum_{f} y^{f}\right)$, is zero, regardless of whether $\left(p,\left\{\left\{x^{h}\right\},\left\{y^{f}\right\}\right\}\right)$ constitutes an equilibrium, so long as consumers exhaust their income (i.e., $p \cdot x^{h}=I^{h}$ ). In other words, Walras' Law holds for the ordinary competitive mechanism when preferences are strictly monotone (more of any good is strictly better). It is clear, however, that Walras’ Law generally fails for the second example. Although assumptions guaranteeing that the Law holds are usually invoked to prove existence theorems, we shall now show that a rather weaker condition will suffice. This observation will enable us to establish an existence theorem for the second example.

Given a GCM, let $Z(\cdot)$ be the corresponding excess demand correspondence.

That is, for any prices $p, Z(p)=\left\{z \mid z=\sum_{h}\left(x^{h}-\omega^{h}\right)-\sum_{f} y^{f}\right.$, and $\left\{x^{h}\right\}$ and $\left\{y^{f}\right\}$ are such that, for all $h$,

$$
x^{h} \text { maximizes } \succsim^{h} \text { subject to } p \cdot x^{h} \leq I^{h}\left(p,\left\{y^{f}\right\}\right)
$$

and, for all $f$,

$y^{f}$ maximizes firm $f$ s profit in $Y^{f}$ given prices $\left.p\right\}$.

The following is our basic existence result:

Lemma (Existence): Given a GCM, suppose that $Z(\cdot)$ is well defined, upper hemicontinuous, and convex- and compact-valued. Suppose that if $p$ is such that $p_{i}=0$ for some $i$, then for all $z \in Z(p), z_{i}>0$. Finally, suppose that, for all $p$ and all $z \in Z(p)$, 
either (a) $z=0$ or (b) there exist $i$ and $j$ such that $z_{i}>0$ and $z_{j} \leq 0$. (Note that this last hypothesis constitutes a weakening of Walras’ Law). Then, there exists an equilibrium. ${ }^{3}$ Proof: Because $Z(\cdot)$ is upper hemi-continuous and, for any $p$ and $i, z_{i}>0$ if $z \in Z(p)$ and $p_{i}=0$, there exists $\delta>0$ such that, for all $p$, all $i$ and all $z \in Z(p), z_{i}>0$ if $p_{i}<\delta$. Hence, upper hemi-continuity implies that there exists $K>0$ such that for all $p$, all $z \in Z(p)$, and all $j, z_{j}+K p_{j}>0$. Define the correspondence

$$
H(p)=\left\{h \mid h=\frac{z+K p}{\sum_{i}\left(z_{i}+K p_{i}\right)}, z \in Z(p)\right\} .
$$

The correspondence $H(\cdot)$ takes the unit simplex to itself. It is upper hemi-continuous and convex- and compact-valued because $Z(\cdot)$ is. Therefore, by the Kakutani fixed point lemma, there exists $\bar{p}$ such that $\bar{p} \in H(\bar{p})$. Choose $\bar{Z} \in Z(\bar{p})$ such that.

$$
\bar{p}=\frac{\bar{z}+K \bar{p}}{\sum_{i}\left(z_{i}+K \bar{p}_{i}\right)} .
$$

If $\bar{p}_{j}=0$ for some $j$, then, by hypothesis, $\bar{z}_{j}>0$, which contradicts $(*)$. Hence, $\bar{p}_{j}>0$ for all $j$. Thus, if $\sum_{i} \bar{Z}_{i}>0$, then from $(*), \bar{z}_{j}>0$ for all $j$, contradicting our weakened Walras' Law. Similarly, if $\sum_{i} \bar{Z}_{i}<0$, then $(*)$ implies that $\bar{Z}_{j}<0$ for all $j$, also a contradiction. Therefore, $\sum_{i} \bar{z}_{i}=0$, and so, from $(*), \bar{z}_{j}=0$ for all $j$, i.e., $\bar{p}$ and the allocation corresponding to $\bar{Z}$ constitute an equilibrium.

Q.E.D.

\footnotetext{
${ }^{3}$ Varian (1981) makes a related observation for excess demand functions.
} 


\section{Application of the Existence Theorem}

Theorem 1 (Existence of equilibrium at a Pareto efficient allocation): Let the allocation $\left\{\left\{\tilde{x}^{h}\right\},\left\{\tilde{y}^{f}\right\}\right\}$ be Pareto efficient, and suppose that, for all $h$, all components of $\tilde{x}^{h}$ are strictly positive. ${ }^{4}$ Suppose that, for all $h$ and $p, I^{h}\left(p,\left\{y^{f}\right\}\right)=p \cdot \tilde{x}^{h}$. Assume that preferences are convex, continuous, and strictly monotone, and that production sets are convex, closed, and bounded. ${ }^{5}$ Then an equilibrium exists.

Proof: Because the aggregate feasible set $\left(\sum_{h} \omega^{h}+\sum_{f} Y^{f}\right) \cap \mathbb{R}_{+}^{\ell}$ is bounded, we can choose $M$ big enough so that if each consumer $h$ is limited to the truncated consumption set $\mathbb{R}_{+}^{\ell} \cap[-M, M]^{\ell}$, then any allocation $\left\{\left\{x^{h}\right\},\left\{y^{f}\right\}\right\}$ for which, for some $h, x^{h}$ is on the truncation boundary must be infeasible. Let $Z(\cdot)$ be the excess demand correspondence for the truncated consumption sets. It is well-defined, upper hemi-continuous, and compact-valued because agents' (i.e., consumers' and firms') objectives are continuous, their choice sets are closed and bounded, and the components of each $\tilde{x}^{h}$ are strictly positive. It is convex-valued because agents' objectives and their choice sets are convex.

Consider $p$ such that $p_{i}=0$ for some $i$. Choose $z \in Z(p)$ and write $z=\sum_{h}\left(x^{h}-\omega^{h}\right)-\sum_{f} y^{f}$. Because preferences are strictly monotone, $x_{i}^{h}=M$ for all $h$. Hence, $z_{i}>0$.

\footnotetext{
${ }^{4}$ This hypothesis can be relaxed using standard methods, as in Debreu (1959).

${ }^{5}$ The boundedness assumption can be dropped if we impose certain conditions on the aggregate production set $\sum_{f} Y^{f}$; see Debreu (1959).
} 
Thus, to apply our Lemma to the truncated economy, it remains only to verify that $Z(\cdot)$ satisfies our weak Walras' Law. Suppose, for given $p$ and $\bar{Z} \in Z(p)$, that $\bar{Z} \neq 0$. Write $\bar{z}=\sum_{h}\left(\bar{x}^{h}-\omega^{h}\right)-\sum_{f} \bar{y}^{f}$. By definition of the GCM, $p \cdot \bar{x}^{h} \leq p \cdot \tilde{x}^{h}$ for all $h$. From profit maximization $p \cdot \bar{y}^{f} \geq p \cdot \tilde{y}^{f}$ for all $f$. Therefore, $p \cdot \bar{z} \leq p \cdot\left(\sum_{h}\left(\tilde{x}^{h}-\omega^{h}\right)-\sum_{f} \tilde{y}^{f}\right)=0$, where the last equation holds because $\left\{\left\{\tilde{x}^{h}\right\},\left\{\tilde{y}^{f}\right\}\right\}$ is Pareto efficient and preferences are strictly monotone. Thus, because $\bar{z} \neq 0$, there exists good $j$ such that $\bar{z}_{j}<0$. If $\bar{z} \leq 0$, then the allocation $\left\{\left\{\bar{x}^{h}\right\},\left\{\bar{y}^{f}\right\}\right\}$ is feasible. Because consumer $h$ can afford $\tilde{x}^{h}, \bar{x}^{h} \succsim^{h} \tilde{x}^{h}$. Hence, $\left\{\left\{\bar{x}^{h}\right\},\left\{\bar{y}^{f}\right\}\right\}$ is a Pareto efficient allocation with excess supply in good $j$, a contradiction of strictly monotone preferences. Thus, there exists $i$ such that $\bar{z}_{i}>0$ and so all the hypotheses of the Lemma hold. Hence, there exists an equilibrium $\hat{p},\left\{\left\{\hat{x}^{h}\right\},\left\{\hat{y}^{f}\right\}\right\}$ when consumers are confined to their truncated consumption sets. Because the allocation is feasible, no $\hat{x}^{h}$ can lie on the truncation boundary. Suppose, for consumer $h$, there exists $\hat{\hat{x}}^{h}$ outside his truncated consumption set such that he strictly prefers $\hat{\hat{x}}^{h}$ to $\hat{x}^{h}$ and $\hat{p} \cdot \hat{\hat{x}} \leq \hat{p} \cdot \tilde{x}^{h}$. But then strict monotonicity and preference convexity imply that any strict convex combination of $\hat{\hat{x}}^{h}$ and $\hat{x}^{h}$ is strictly preferred to $\hat{x}^{h}$, which implies that there exists a consumption bundle in the truncated consumption set strictly preferred to $\hat{x}^{h}$, a contradiction. We conclude that, for all $h, \hat{x}^{h}$ globally maximizes consumer $h$ 's preferences subject to his budget constraint. That is, $\hat{p},\left\{\left\{\hat{x}^{h}\right\},\left\{\hat{y}^{f}\right\}\right\}$ is a full-fledged equilibrium. 
Q.E.D.

\section{The Welfare Theorems}

The first welfare theorem asserts that a competitive equilibrium is Pareto efficient. The natural generalization to our framework is the following

Theorem 2: (First Welfare Theorem): If preferences are strictly monotone, then any equilibrium of a GCM is Pareto efficient.

Proof: Suppose that prices $\hat{p}$ and allocation $\left\{\left\{\hat{x}^{h}\right\},\left\{\hat{y}^{f}\right\}\right\}$ constitute an equilibrium of a GCM. Suppose, contrary to the Theorem, there exists a feasible allocation $\left\{\left\{x^{h}\right\},\left\{y^{f}\right\}\right\}$ that Pareto dominates $\left\{\left\{\hat{x}^{h}\right\},\left\{\hat{y}^{f}\right\}\right\}$. By definition of equilibrium and from strictly monotone preferences, we have

$$
\sum_{h} x^{h}=\sum_{h} \omega^{h}+\sum_{f} y^{f} \text { and } \sum_{h} \hat{x}^{h}=\sum_{h} \omega^{h}+\sum_{f} \hat{y}^{f}
$$

and

$$
\hat{p} \cdot x^{h} \geq \hat{p} \cdot \hat{x}^{h} \text { for all } h
$$

with strict inequality for those consumers $h$ who strictly prefer $x^{h}$ to $\hat{x}^{h}$. Thus, summing (2) across consumers, we obtain

$$
\sum_{h} \hat{p} \cdot x^{h}>\sum_{h} \hat{p} \cdot \hat{x}^{h}
$$

From profit maximization, we have $\hat{p} \cdot y^{f} \leq \hat{p} \cdot \hat{y}^{f}$ for all $f$, and so

$$
\sum_{f} \hat{p} \cdot y^{f} \leq \sum_{f} p \cdot \hat{y}^{f}
$$

Subtracting (4) from (3) and also subtracting endowments, we obtain 


$$
\hat{p} \cdot\left(\sum_{h}\left(\hat{x}^{h}-\omega^{h}\right)-\sum_{f} \hat{y}^{f}\right)<\hat{p} \cdot\left(\sum_{h}\left(x^{h}-\omega^{h}\right)-y^{f}\right),
$$

which contradicts (1). Thus, the equilibrium allocation is Pareto efficient after all.

Q.E.D.

The proof of Theorem 2 will be recognized as identical to that usually given for the first welfare theorem. We have included it here only for comparison with the proof of the decentralization theorem:

Theorem 3: (Decentralization of a Pareto efficient allocation): Assume that preferences are strictly monotone. Suppose that allocation $\left\{\left\{\tilde{x}^{h}\right\},\left\{\tilde{y}^{f}\right\}\right\}$ is Pareto efficient. Consider the GCM in which, given prices $p$, consumer $h$ receives income $I^{h}=p \cdot \tilde{X}^{h}$. Then, if an equilibrium of this GCM exists, $\left\{\left\{\tilde{x}^{h}\right\},\left\{\tilde{y}^{f}\right\}\right\}$ is an equilibrium allocation.

Proof: Suppose that $\hat{p}$ is an equilibrium price vector and $\left\{\left\{\hat{x}^{h}\right\},\left\{\hat{y}^{f}\right\}\right\}$ is a corresponding equilibrium allocation for the GCM described. From Theorem 2, $\left\{\left\{\hat{x}^{h}\right\},\left\{\hat{y}^{f}\right\}\right\}$ is Pareto efficient. Therefore, because $\left\{\left\{\tilde{x}^{h}\right\},\left\{\tilde{y}^{f}\right\}\right\}$ is also Pareto efficient and consumer $h$ can afford $\tilde{X}^{h}$, he must be indifferent between $\tilde{X}^{h}$ and $\hat{x}^{h}$, and so, from preference monotonicity, $\hat{p} \cdot \hat{x}^{h}=\hat{p} \cdot \tilde{x}^{h}$. Because firms are profit maximizing, $\hat{p} \cdot \hat{y}^{f} \geq \hat{p} \cdot \tilde{y}^{f}$ for all $f$. If, for some firm $f$, the inequality is strict, then $\hat{p} \cdot \sum_{f} \hat{y}^{f}>\hat{p} \cdot \sum_{f} y^{f}$, and so

$$
\hat{p} \cdot\left(\sum_{h} \hat{x}^{h}-\sum_{f} \hat{y}^{f}\right)<\hat{p} \cdot\left(\sum_{h} \tilde{x}^{h}-\sum_{f} \tilde{y}^{f}\right) .
$$


But, since $\left\{\left\{\tilde{x}^{h}\right\},\left\{\tilde{y}^{f}\right\}\right\}$ and $\left\{\left\{\hat{x}^{h}\right\},\left\{\hat{y}^{f}\right\}\right\}$ are both Pareto efficient and preferences are strictly monotone, $\sum_{h} \tilde{x}^{h}-\sum_{f} \tilde{y}^{f}=\sum_{h} \hat{x}^{h}-\sum_{f} \hat{y}^{f}=\sum_{h} \omega^{h}$, contradicting (6). Thus, $\hat{p} \cdot \hat{y}^{f}=\hat{p} \cdot \tilde{y}^{f}$ for all $f$. Since consumers and firms both are indifferent between the hatted and the tildaed allocations, we conclude that $\left\{\left\{\tilde{x}^{h}\right\},\left\{\tilde{y}^{f}\right\}\right\}$ is itself an equilibrium allocation.

Q.E.D.

The proof of Theorem 3, like that of Theorem 2, is a simple revealed preference argument: given that existential problems can be ignored, agents stay at the pre-trade allocation unless they can make themselves better off. But if the pre-trade allocation is Pareto efficient, improvement is impossible. It is worth emphasizing that Theorem 3 requires no convexity assumptions. The theorem illustrates that convexity in decentralization theorems is needed only to show that equilibrium exists; it is not required to show that the equilibrium occurs at the Pareto efficient allocation. Indeed, it follows directly that if a Pareto efficient allocation cannot be supported as an equilibrium, then starting at this allocation, no equilibrium can exist.

Finally, if existence can be guaranteed without the use of convexity — as in large nonatomic economies-Theorem 3 ensures that Pareto efficient allocations can be decentralized.

The usual second welfare theorem follows immediately from Theorems 1 and 3 :

Theorem 4: (Second Welfare Theorem): Suppose that preferences and production sets satisfy the hypotheses of Theorem 1. Then, if $\left\{\left\{\tilde{x}^{h}\right\},\left\{\tilde{y}^{f}\right\}\right\}$ is Pareto efficient and, for all $h$, all components of $\tilde{x}^{h}$ are strictly positive, there exist prices $\hat{p}$ and balanced transfers 
$\left\{T^{h}\right\}$ (i.e., summing to zero) such that $\left\{\left\{\tilde{x}^{h}\right\},\left\{\tilde{y}^{f}\right\}\right\}$ is an equilibrium allocation with respect to the mechanism that, for each $p$, gives consumer $h$ the income $p \cdot \omega^{h}+\sum_{f} \theta_{f}^{h} p \cdot \tilde{y}^{f}+T^{h}$

Proof: Under the hypotheses, Theorem 1 implies the existence of an equilibrium of the GCM in which, for each $p$, consumer $h$ is assigned income $p \cdot \tilde{x}^{h}$. Theorem 3 then implies that, together some price vector $\hat{p},\left\{\left\{\tilde{x}^{h}\right\},\left\{\tilde{y}^{f}\right\}\right\}$ is such an equilibrium. To complete the argument, set $T^{h}=\hat{p} \cdot \tilde{x}^{h}-\left(\hat{p} \cdot \omega+\sum \theta_{f}^{h} \hat{p} \cdot \tilde{y}^{f}\right)$.

Q.E.D.

\section{4. $\quad$ Related Literature}

Our weakening of Walras’ Law_-which replaces the usual equation with an ordinal condition on excess demand — is not the only relaxation of the Law that has been considered in the literature. Specifically, although the strict version of Walras' Law holds in the classical Arrow-Debreu-McKenzie general equilibrium model, one must replace equality with inequality —in models with an infinite commodity space: Aliprantis and Brown (1983), Yannelis (1985) and Podczeck (1997) present existence theorems for this case, and Krasa and Yannelis (1994) establish existence using this same version of the Law in a finite commodity space.

Similarly, we are not the first to draw a connection between existence and the second welfare theorem. Indeed, the classic example of Arrow (1951), showing that the second theorem may fail when the sum of endowments is not in the interior of the aggregate consumption set, also illustrates equilibrium non-existence. When both the commodity space and the price space are vector lattices, Mas-Colell and Richard (1986) 
show that restrictions on preferences ensuring existence are identical to those required for the second welfare theorem. For further discussion and analysis of the second welfare theorem in a variety of contexts, see Aliprantis et al (2001) and Aliprantis et al (2002).

\section{Concluding Remarks}

This paper has reconsidered the principal theorems of general equilibrium theory. We have argued that:

(A) There are interesting general equilibrium models in which Walras' Law fails to hold out of equilibrium.

(B) Equilibrium may nevertheless exist in these models because a weaker version of Walras’ Law holds.

(C) If existence is taken for granted, the second welfare theorem-just like the firstfollows from a simple revealed preference analysis. The usual statement of the second theorem involves an existential claim that is the reason behind its mathematical "difficulty." Separation of the theorem into two parts—-the existence part invoking the weakened Walras' Law—makes clear that convexity conditions may be needed for existence but not for the part of the theorem constituting its real substance. 


\section{References}

1. Aliprantis, C.D., Brown, D.J.: Equilibria in Markets with a Riesz Space of Commodities. Journal of Mathematical Economics 11, 189-207 (1983)

2. Aliprantis, C.D., Cornet, B., Tourky, R.: Economic Equilibrium: Optimality and Price Decentralization. Positivity 6, 205-241 (2002)

3. Aliprantis, C.D., Tourky, R., Yannelis, N.C.: A Theory of Value with Non-linear Prices: Equilibrium Analysis Beyond Vector Lattices. Journal of Economic Theory 100, 22-72 (2001)

4. Arrow, K.J.: An Extension of the Basic Theorems of Welfare Economics, Proceedings of the Second Berkeley Symposium on Mathematical Statistics and Probability II, Part 1. Berkeley: University of California Press (1951)

5. Debreu. G.: Theory of Value. New Haven: Yale University Press (1959)

6. Krasa, S., Yannelis, N.C.: An Elementary Proof of the Knaster-KuratowskiMazurkiewitcz-Shapley Theorem. Economic Theory 4, 467-471 (1994)

7. Mas-Colell, A., Richard, S.F.: A New Approach to the Existence of Equilibria in Vector Lattices. Journal of Economic Theory 53, 1-11 (1986)

8. Podczeck, K.:Markets with Infinitely Many Commodities and a Continuum of Agents with Non-Convex Preferences. Economic Theory 9, 385-426 (1997)

9. Varian, H.: Dynamical Systems with Applications to Economics. Handbook of Mathematical Economics. vol. I, Amsterdam: North Holland (1981)

10. Varian, H.: Microeconomic Analysis. Third Edition, New York: Norton (1992) 
11. Yannelis, N.C.: On a Market Equilibrium Existence Theorem with an Infinite Number of Commodities. Journal of Mathematical Analysis and Applications 108, 595-599 (1985) 